\title{
Efficacy and safety of additional surgery after non-curative endoscopic submucosal dissection for early colorectal cancer
}

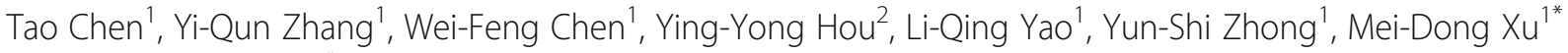 \\ and Ping-Hong Zhou ${ }^{1 *}$
}

\begin{abstract}
Background: Additional surgery is recommended when early colorectal cancer (ECRC) is resected by non-curative endoscopic submucosal dissection (ESD) and there is significant risk of lymph node metastasis (LNM). The aim of this study was to investigate the efficacy and safety of additional surgery after non-curative ESD for ECRC and evaluate long-term outcomes.
\end{abstract}

Methods: Patients with ECRC who underwent ESD and additional surgery between July 2007 and November 2013 were identified. Histology and patient data were collected during an average period of more than 5 years to determine tumor stage and type, resection status, complications, tumor recurrence, and distant metastasis.

Results: Fifty-one patients who underwent additional surgery were eligible for analysis. Overall, regional LNM was detected in 5 patients (9.8\%) and presence of lymphovascular infiltration was a significant risk factor. Surgery-related complications occurred in 3 patients (5.9\%). During a median follow-up period of 59 months, no metastasis or local recurrence was observed. Three patients died of other diseases and no CRC-related deaths took place.

Conclusions: Additional surgery after non-curative ESD for ECRC is effective and safe and should be encouraged to foster curative treatment and better long-term outcomes.

Keywords: Early cancer, Colorectum, Endoscopic submucosal dissection, Additional surgery

\section{Background}

Endoscopic submucosal dissection (ESD) is the standard treatment for early colorectal cancers (ECRCs) that have negligible risk of lymph node metastasis (LNM) [1]. Studies have reported that ESD provides high en bloc resection rates, which contributes to accurate histopathological evaluation $[2,3]$. In addition, ESD provides high $\mathrm{R} 0$ resection rates to minimize risk of recurrence. However, curative resection can be evaluated successful only after the patient meets the pathological low-risk criteria, including submucosal invasion of less than $1000 \mu \mathrm{m}$, exclusion of poor differentiation, exclusion of lymphovascular infiltration, and no tumor budding [4]. When one

\footnotetext{
* Correspondence: zsxumeidong@yahoo.com; pinghongzhou@yahoo.com ${ }^{1}$ Endoscopy center, Zhongshan Hospital, and Endoscopy Research Institute, Fudan University, Shanghai 200032, China

Full list of author information is available at the end of the article
}

of these criteria is not met, additional surgery with lymph node dissection is recommended to prevent lymph node or distant metastasis. To date, few data are available regarding the safety of additional surgery after non-curative ESD and the long-term outcomes. Thus, we investigated the efficacy and safety of additional surgery after non-curative ESD for ECRC and examined its long-term outcomes with an average period of more than 5 years.

\section{Methods \\ Study population and data collection \\ Between July 2007 and November 2013, a total of 51 ECRCs in 51 patients who had additional surgery after non-curative ESD were identified and analyzed in this study. ESD is indicated for lesions requiring endoscopic en bloc resection for which it is difficult to use the snare}


technique, including laterally spreading tumor (LST), superficial invasive submucosal cancer, large depressed tumors, and large elevated lesions that are likely malignant. Curative resection is defined as submucosal invasion of less than $1000 \mu \mathrm{m}$ with exclusion of poor differentiation, exclusion of lymphovascular infiltration, and no tumor budding [4]. In addition, piecemeal resection is the critical risk factor for local recurrence. When one of these criteria is not met, additional surgery is recommended. The study was performed in accordance with the Declaration of Helsinki. All patients were informed of the benefits of ESD and the risks of additional surgery, and each patient provided written informed consent for the procedure. This study protocol was approved by the Institutional Review Board of Zhongshan Hospital (No.2009135).

\section{Treatment for ECRC}

ESDs were performed with patients under intravenous anesthesia. Additional surgeries were performed with patients under general anesthesia. Indices of the patients' cardiorespiratory functions (heart rate, blood pressure, and oxygen saturation) were continually monitored. Endoscopic equipment and accessories were introduced in previous reports $[5,6]$. Some colorectal tumors were resected using a combined endoscopic mucosal resection (EMR) and ESD technique (hybrid ESD), with a special ESD knife, and resection was completed by snaring [7]. Some degree of submucosal dissection was involved in hybrid ESD after marginal pre-cutting [1]. When ESD was revealed to be non-curative, patients were referred to surgeons for consultation. For patients who agreed, additional radical surgery was performed in the same manner as initial standard surgery.

\section{Histopathological evaluation}

Resected specimens were stretched, pinned, fixed in formalin solution, and assessed microscopically. The WHO classification for tumors of the digestive system was used for histopathological evaluation [8]. An en bloc resection was defined as an excision of the tumor in one piece without fragmentation. Histologic complete resection was defined as en bloc resection with negative vertical and lateral margins. Curative resection was defined as a histologic complete resection with no risk of LNM as indicated by histopathological examination of the resected specimen according to the criteria issued by the Japanese Society for Cancer of the Colon and Rectum (JSCCR) [9].

\section{ESD-related complications}

Minor bleeding occurred in most cases. It was treated with immediate coagulation. Delayed bleeding was defined as hematochezia or melena requiring an endoscopic hemostatic procedure from 0 to 14 days after ESD completion. Perforation was classified as a colorectal wall penetration detected during or after ESD procedure.

\section{Follow-up}

All patients were followed up either at our institution or in partnership with their referring institutions. Followup colonoscopy was performed at 6 and 12 months after the operation and then annually in 5 years. Abdominal Computed Tomography (CT) was carried out annually. To analyze long-term outcomes, a questionnaire was sent to patients who had no follow-up medical records at our institution or at our partner centers.

\section{Statistical analysis}

Clinicopathological and endoscopic data, including age, sex, tumor characteristics, histology, resection rates, complications, local recurrence, metastasis, and survival rates were collected and analyzed. Statistical analysis was performed using a chi-squared test or Fisher's exact test as appropriate. Commercial software (IBM SPSS Statistics 18; SPSS, Chicago, IL, U.S.) was used for statistical analysis $(P<0.05$ was considered statistically significant).

\section{Results}

Baseline characteristics of patients and tumors

In this retrospective study, we identified and analyzed 51 ECRC treated by ESD in 51 patients, including 31 men and 20 women. The mean age of the patients was $63.0 \pm$ 9.5 years; $56.9 \%(29 / 51)$ of the patients were older than 60 years. The mean tumor size was $2.6 \pm 0.9 \mathrm{~cm}$ (median, $2.0 \mathrm{~cm}$; range, 1.5-5.0 cm). Among these tumors, 42 tumors $(82.4 \%)$ were smaller than $4.0 \mathrm{~cm}$, and the other 9 tumors $(17.6 \%)$ were larger than $4.0 \mathrm{~cm}$. Tumor location included 7 in the right colon (13.7\%), 14 in the left colon (27.4\%), and 30 in the rectum (58.9\%). Macroscopic types included 31 polypoid tumors $(60.8 \%)$ and 20 LSTs (39.2\%). Patient and tumors details are shown in Table 1.

\section{Outcomes of ESD for ECRCs}

Resection methods included 41 cases of ESD (80.4\%) and 10 hybrid ESD (19.6\%). En bloc resection was achieved in 46 of the 51 treated tumors (90.2\%), histological complete resection was achieved in 34 cases (66.7\%). Histologically, of the 51 tumors, there were 15 (29.4\%) T1 cancers with superficial submucosal invasion $(<1000 \mu \mathrm{m})$ and $36(70.6 \%)$ T1 cancers with deep submucosal invasion $(\geq 1000 \mu \mathrm{m})$. Specifically, there were 5 cancers $(9.8 \%)$ with invasive lymphovascular infiltration. In the present study, 2 patients had perforations that were closed by clips during the ESD procedure and no patient had delayed bleeding. There were no treatmentrelated deaths. Outcomes related to ESD for ECRCs are shown in Table 2. 
Table 1 Baseline characteristics of the patient and tumors

\begin{tabular}{ll}
\hline Variables & Total \\
\hline Patients & 51 \\
Age & \\
$\quad$ Average \pm s.d.(median, range),years & $63.0 \pm 9.5(63,38-80)$ \\
$\quad \leq 60$ years & $22(43.1 \%)$ \\
$\quad>60$ years & $29(56.9 \%)$ \\
Sex & \\
Male & $31(60.8 \%)$ \\
Female & $20(39.2 \%)$ \\
Tumors & 51 \\
Tumor size & \\
$\quad$ Average \pm s.d.(median, range),cm & $2.6 \pm 0.9(2.0,1.5-5.0)$ \\
$\quad<40$ mm & $42(82.4 \%)$ \\
$\quad \geq 40$ mm & $9(17.6 \%)$ \\
Tumor location & \\
Right side of colon & $7(13.7 \%)$ \\
Left side of colon & $14(27.4 \%)$ \\
Rectum & $30(58.9 \%)$ \\
Growth type & \\
Laterally spreading tumor & $20(39.2 \%)$ \\
Polypoid & $31(60.8 \%)$ \\
\hline
\end{tabular}

\section{Outcomes of additional surgery after colorectal ESD}

The average hospital stay after additional surgery was $7.4 \pm 3.1$ days (median, 6; range, 4-21). There was 1 patient with postoperative heart failure, 1 patient with postoperative anemia, and 1 patient with postoperative urinary tract infection. Pathological examination after additional surgery found 5 patients with regional LNM (9.8\%). In this study, lost to follow-up occurred in 2 patients (3.9\%) and the follow-up rate was 96.1\% (49/51). The overall median follow-up period was 59 months (average 63.9, range 30-115 months). During the followup period, no local recurrence was detected. Furthermore, no patients developed metastasis to either the lymph nodes or distant organs. Three patients died of other diseases and no CRC-related death was found. These data appear in Table 3.

\section{Association of the clinicopathological characteristics of ECRCs with regional LNM}

In this study, reasons for additional surgery after ESD for ECRCs included incomplete resection (17 cases, $33.3 \%$ ), positive margin (13 cases, $25.5 \%$ ), T1 cancers ( $\geq 1000 \mu \mathrm{m}, 34$ cases, $66.7 \%$ ), lymphovascular infiltration (5 cases, 9.8\%), and poor differentiation (2 cases, $3.9 \%$, Table 4). Of note, 2 patients who had lymphovascular infiltration had deep T1 cancers and 1 patient had positive margin. On univariate analysis,
Table 2 Outcomes of ESD for 51 early colorectal cancers

\begin{tabular}{ll}
\hline Variables & Total \\
\hline Number of tumors & 51 \\
Resection Method & \\
ESD & $41(80.4 \%)$ \\
Hybrid ESD & $10(19.6 \%)$ \\
Resection status & \\
En bloc & $46(90.2 \%)$ \\
Piecemeal & $5(9.8 \%)$ \\
Histological complete resection & \\
Complete & $34(66.7 \%)$ \\
Incomplete & $17(33.3 \%)$ \\
Histology & \\
T1 cancer $(<1000 \mu m)$ & $15(29.4 \%)$ \\
T1 cancer $(\geq 1000 \mu m)$ & $36(70.6 \%)$ \\
Differentiation & \\
Well and moderate & $49(96.1 \%)$ \\
Poor & $2(3.9 \%)$ \\
Lymphovascular infiltration & \\
Absence & \\
Presence & \\
Complications & $46(90.2 \%)$ \\
Delayed bleeding & $5(9.8 \%)$ \\
\hline & \\
\hline
\end{tabular}

Table 3 Outcomes of additional surgery after colorectal ESD

\begin{tabular}{ll}
\hline Variables & Total \\
\hline Number of patients & 51 \\
Postoperative hospital stay & \\
Average \pm s.d.(median, range), day & $7.4 \pm 3.1(6,4-21)$ \\
Complications & $3(5.9 \%)$ \\
Postoperative heart failure & $1(2.0 \%)$ \\
Anemia & $1(2.0 \%)$ \\
Urinary tract infection & $1(2.0 \%)$ \\
Pathological examination & \\
Lymph node metastasis & $5(9.8 \%)$ \\
Follow up & \\
Lost to follow up & $2(3.9 \%)$ \\
Average(median, range), month & $63.9(59,30-115)$ \\
Recurrence & $0(0.0 \%)$ \\
Metastasis & $0(0.0 \%)$ \\
Overall survival & $48 / 51(94.1 \%)$ \\
\hline
\end{tabular}


Table 4 Reasons of additional surgery after ESD

\begin{tabular}{ll}
\hline Variables & Total \\
\hline Number of tumors & 51 \\
Piecemeal resection & $5(9.8 \%)$ \\
Positive margin & $13(25.5 \%)$ \\
$\quad$ Vertical & $11(21.6 \%)$ \\
$\quad$ Circumferential & $2(3.9 \%)$ \\
T1 cancer $(\geq 1000 \mu m)$ & $34(66.7 \%)$ \\
Poor differentiation & $2(3.9 \%)$ \\
Lymphovascular infiltration & $5(9.8 \%)$ \\
\hline
\end{tabular}

presence of lymphovascular infiltration had significant association with LNM in patients with ECRCs treated by non-curative ESD $(P=0.005)$. No other clinicopathological characteristics were found to be associated with regional LNM (Table 5).

\section{Discussion}

Additional surgery is recommended when ECRCs are resected by non-curative ESD and LNM risk exists. Sometimes, additional surgery after endoscopic resection (ER) was reported not to be performed because of high surgical risk including comorbidities and old age [10, 11]. The present study showed that additional surgery after non-curative ESD for ECRCs was safe. Postoperative complications occurred only in 3 patients (5.9\%). All of the patients with complications were successfully taken care of using conservative treatments and there were no perioperative deaths. In this study, regional LNM was observed in 5 patients $(9.8 \%)$. Compared the risk of LNM to the rate of complications, patients benefitted from additional surgery after non-curative ESD. The average postoperative hospital stay was 7.4 days that was comparable to direct surgery. The long-term outcomes were also excellent and no patients developed metastasis to either the lymph nodes or distant organs. The overall survival rate was up to $94.1 \%$ and no CRCrelated deaths took place during follow-up, which lasted more than 5 years on average.

Currently, indications for ER of early gastrointestinal epithelial cancers have been expanded when no risk of LNM exists. Patients with T1-stage ECRC have LNM in $6-16 \%$ of cases, which means that most patients with ECRC do not have metastasis [12]. However, when LNM exists, the cancer cannot be curatively resected by ER alone and requires lymphadenectomy. In addition, piecemeal resection is the critical risk factor for local recurrence, regardless of the ER method used [1]. National Comprehensive Cancer Network (NCCN) guidelines recommend follow-up for T1-stage CRC following ER in a single specimen (en bloc), negative vertical and lateral resection margins, histological grade 1 or 2 , and without
Table 5 The association of the clinicopathological characteristics of 51 early colorectal cancers with lymph node metastasis

\begin{tabular}{|c|c|c|}
\hline Factors & Lymph node metastasis & $P$ value \\
\hline \multicolumn{3}{|l|}{ Age } \\
\hline$\leq 60$ years & $3 / 22(13.6 \%)$ & \multirow[t]{2}{*}{0.638} \\
\hline$>60$ years & $2 / 29(6.9 \%)$ & \\
\hline \multicolumn{3}{|l|}{ Sex } \\
\hline Male & $3 / 31(9.7 \%)$ & \multirow[t]{2}{*}{1.000} \\
\hline Female & $2 / 20(10.0 \%)$ & \\
\hline \multicolumn{3}{|l|}{ Tumor size } \\
\hline$<40 \mathrm{~mm}$ & $5 / 42(11.9 \%)$ & \multirow[t]{2}{*}{0.571} \\
\hline$\geq 40 \mathrm{~mm}$ & $0 / 9(0.0 \%)$ & \\
\hline \multicolumn{3}{|l|}{ Tumor location } \\
\hline Rectum & $4 / 30(13.3 \%)$ & \multirow[t]{2}{*}{0.391} \\
\hline Colon & $1 / 21(4.8 \%)$ & \\
\hline \multicolumn{3}{|l|}{ Growth type } \\
\hline Polypoid & $4 / 31(12.9 \%)$ & \multirow[t]{2}{*}{1.000} \\
\hline LST & $1 / 20(5.0 \%)$ & \\
\hline \multicolumn{3}{|l|}{ Resection Method } \\
\hline ESD & $5 / 41(12.2 \%)$ & \multirow[t]{2}{*}{0.569} \\
\hline Hybrid ESD & $0 / 10(0.0 \%)$ & \\
\hline \multicolumn{3}{|l|}{ Resection status } \\
\hline En bloc & $5 / 46(10.9 \%)$ & \multirow[t]{2}{*}{1.000} \\
\hline Piecemeal & 0/5(0.0\%) & \\
\hline \multicolumn{3}{|c|}{ Histological complete resection } \\
\hline Complete & $4 / 34(11.8 \%)$ & \multirow[t]{2}{*}{0.654} \\
\hline Incomplete & $1 / 17(5.9 \%)$ & \\
\hline \multicolumn{3}{|l|}{ Histology } \\
\hline T1 cancer $(<1000 \mu \mathrm{m})$ & $1 / 15(6.7 \%)$ & \multirow[t]{2}{*}{1.000} \\
\hline T1 cancer $(\geq 1000 \mu \mathrm{m})$ & $4 / 36(11.1 \%)$ & \\
\hline \multicolumn{3}{|l|}{ Differentiation } \\
\hline Well and moderate & $1 / 49(2.0 \%)$ & \multirow[t]{2}{*}{0.078} \\
\hline Poor & $1 / 2(50 \%)$ & \\
\hline \multicolumn{3}{|l|}{ Lymphovascular infiltration } \\
\hline Absence & $2 / 46(4.3 \%)$ & \multirow[t]{2}{*}{0.005} \\
\hline Presence & 3/5(60.0\%) & \\
\hline
\end{tabular}

lymphatic and vascular invasion [13]. Submucosal invasion $\geq 1000 \mu \mathrm{m}$, presence of lymphovascular infiltration, poor differentiation, tumor budding, and incomplete resection are independently associated with increased risk of LNM and residual cancer. Therefore, a radical resection is warranted. In this study, the most common reason for additional surgery after ESD was T1-stage cancer with submucosal invasion $\geq 1000 \mu \mathrm{m}$. Kikuchi et al. divided the submucosa (SM) into SM1, SM2, and SM3 and indicated that the cancer with the depth of 
submucosal invasion had a risk of LNM of $0 \%, 10 \%$, and $25 \%$, respectively [14]. Thus, additional surgery is essential for these patients with SM2 and SM3 cancers $(\geq 1000 \mu \mathrm{m})$.

In the present study, lymphovascular infiltration (5 cases, 9.8\%) was also an important reason for additional surgery after non-curative ESD. Kim and his colleagues reported that lymphovascular infiltration was independently associated with LNM [15]. The association between lymphovascular infiltration and regional LNM is consistent with previous studies of factors related to LNM. However, poor differentiation and submucosal invasion depth were not statistically significantly related to LNM, which was probably due to a relatively small number of cases in this study.

The limitations of this study are its retrospective design and relatively small number of study cases. We also did not compare these values to those of patients who underwent surgery initially after diagnosis. Thus, a prospective, randomized controlled study must be performed in the future to validate the results observed.

\section{Conclusions}

Additional surgery after non-curative ESD for ECRC is effective and safe. Patients who underwent additional surgery had favorable long-term outcomes. Additional surgery should be encouraged after non-curative endoscopic resection to foster curative treatment and better long-term outcomes.

\begin{abstract}
Abbreviations
CT: Computed Tomography; ECRC: Early colorectal cancer; EMR: Endoscopic mucosal resection; ER: Endoscopic resection; ESD: Endoscopic submucosal dissection; JSCCR: Japanese Society for Cancer of the Colon and Rectum; LNM: Lymph node metastasis; LST: Laterally spreading tumor; WHO: Word Health Organization
\end{abstract}

\section{Acknowledgements}

Not applicable.

\section{Funding}

This work was supported by grants from the National Natural Science Foundation of China [grant number, 81502523]; and grant from Shanghai Committee of Science and Technology [grant number, 16DZ2280900 and 17140901100]

\section{Availability of data and materials}

All data generated or analyzed during this study are included in this published article.

\footnotetext{
Authors' contributions

Conception and design of the study: PHZ, MDX. Generation, collection, acquisition, analysis and/or interpretation of data: TC, LQY, YSZ, YQZ, WFC, YYH. Drafting and critical revision of the manuscript: TC, PHZ, MDX. Statistical analysis: TC. Study supervision: PHZ, MDX, TC. Approval of the final version of the manuscript: PHZ, MDX. All authors have read and approved the manuscript.
}

\section{Ethics approval and consent to participate}

The study was performed in accordance with the Declaration of Helsinki. All patients were informed of the benefits of ESD and the risks of additional surgery, and each patient provided written informed consent for the procedure. This study protocol was approved by the Institutional Review Board of Zhongshan Hospital (No.2009135).

Consent for publication

Not applicable.

\section{Competing interests}

The authors declare that they have no competing interests.

\section{Publisher's Note}

Springer Nature remains neutral with regard to jurisdictional claims in published maps and institutional affiliations.

\section{Author details}

${ }^{1}$ Endoscopy center, Zhongshan Hospital, and Endoscopy Research Institute, Fudan University, Shanghai 200032, China. ${ }^{2}$ Department of Pathology,

Zhongshan Hospital of Fudan University, Shanghai 200032, China.

Received: 17 April 2017 Accepted: 21 November 2017

Published online: 28 November 2017

\section{References}

1. Oka S, et al. Local recurrence after endoscopic resection for large colorectal neoplasia: a multicenter prospective study in Japan. Am J Gastroenterol. 2015;110(5):697-707

2. Saito Y, et al. Clinical outcome of endoscopic submucosal dissection versus endoscopic mucosal resection of large colorectal tumors determined by curative resection. Surg Endosc. 2010;24(2):343-52.

3. Saito $Y$, et al. A prospective, multicenter study of 1111 colorectal endoscopic submucosal dissections (with video). Gastrointest Endosc. 2010; 72(6):1217-25.

4. Pimentel-Nunes $P$, et al. Endoscopic submucosal dissection: European Society of Gastrointestinal Endoscopy (ESGE) guideline. Endoscopy. 2015; 47(9):829-54.

5. Chen T, et al. Efficacy and safety of endoscopic submucosal dissection for colorectal Carcinoids. Clin Gastroenterol Hepatol. 2016;14(4):575-81.

6. Saito $Y$, et al. Indications for and technical aspects of colorectal endoscopic submucosal dissection. Gut Liver. 2013;7(3):263-9.

7. Terasaki M, et al. Clinical outcomes of endoscopic submucosal dissection and endoscopic mucosal resection for laterally spreading tumors larger than 20 mm. J Gastroenterol Hepatol. 2012;27(4):734-40.

8. Bosman FT, et al. WHO classification of tumours of the digestive system. Lyon: IARC; 2010.

9. Watanabe T, et al. Japanese Society for Cancer of the colon and Rectum (JSCCR) guidelines 2014 for treatment of colorectal cancer. Int J Clin Oncol. 2015;20(2):207-39.

10. Oda l, et al. Treatment strategy after non-curative endoscopic resection of early gastric cancer. Br J Surg. 2008:95(12):1495-500.

11. Choi JY, et al. Non-curative endoscopic resection does not always lead to grave outcomes in submucosal invasive early gastric cancer. Surg Endosc. 2015;29(7):1842-9.

12. Ricciardi $R$, et al. Population-based analyses of lymph node metastases in colorectal cancer. Clin Gastroenterol Hepatol. 2006;4(12):1522-7.

13. Bartel MJ, Brahmbhatt BS, Wallace MB. Management of colorectal T1 carcinoma treated by endoscopic resection from the western perspective. Dig Endosc. 2016;28(3):330-41.

14. Kikuchi $R$, et al. Management of early invasive colorectal cancer. Risk of recurrence and clinical guidelines. Dis Colon Rectum. 1995;38(12):1286-95.

15. Kim B, et al. The risk of lymph node metastasis makes it unsafe to expand the conventional indications for endoscopic treatment of T1 colorectal cancer: a retrospective study of 428 patients. Medicine (Baltimore). 2016; 95(37):e4373. 